\title{
Más programas propedéuticos en Chile. El discurso de los estudiantes en la Universidad Católica Silva Henríquez*
}

\author{
More Propaedeutic Programs in Chile. The discourse of students \\ at Universidad Católica Silva Henríquez \\ Mais Programas propedêticos no Chile: $O$ discurso dos estudantes \\ da Universidade Católica Silva Henríquez.
}

\section{Carola Román Pérez}

Universidad Católica Silva Henríquez, Programa de Bachillerato en Ciencias y Humanidades y Propedéutico. Correo electrónico: groman@ucsh.cl

\begin{abstract}
RESUMEN
Las dificultades de ingreso a la educación superior chilena afectan, especialmente, a los estudiantes que provienen de contextos vulnerables. Esta situación merece un análisis profundo, pues exiten en el sistema educativo jóvenes que se destacan por obtener altas calificaciones a lo largo de los estudios secundarios, sin embargo, producto de la segregación que existe en la educación chilena, es probable que los puntajes que obtengan en la Prueba de Selección Universitaria (PSU), no sean lo suficientemente altos para ingresar a la universidad. Para ellos, algunas instituciones han implementado los Programas propedéuticos. Pero, ¿cuál es su discurso con respecto a estos Programas? ¿Por qué obtienen buenos rendimientos? A través de un análisis estructural de discurso, se busca evidenciar las características que estos jóvenes presentan, las que, con los apoyos necesarios, podrían hacer de ellos verdaderos candidatos para culminar con éxito una carrera universitaria.
\end{abstract}

Palabras clave: programas propedéuticos, PSU, ranking, retención, deserción.

\begin{abstract}
Difficulties when joining Chilean higher education affect, in particular, those students coming from vulnerability contexts within society. This situation deserves a deep analysis since, in the educational system, there are youngsters who have an outstanding performance and obtain high grades through their secondary education but whose scores in the Test of University Selection (PSU), due to the segregation existing in the Chilean education, would not be high enough to enter university. Some institutions have implemented Propaedeutic Programs directed to those students. Thus, some questions arise: What is their point of view towards these Programs? Why do they have good performances? Throughout a structural analysis of discourse, it is intended to show evidence of the characteristics that, with the necessary support, could make these students real candidates to end their university studies successfully.
\end{abstract}

Key words: propaedeutic programs, PSU, ranking, retention, school dropout.

\section{RESUMO}

As dificuldades de ingresso na Educação Superior chilena afetam, especialmente, os estudantes oriundos de contextos vulneráveis. Esta situação merece uma análise profunda, pois há neste sistema educativo, jovens que se destacam por obter altas notas ao longo do Ensino Secundário, mas que, por causa da desigualdade existente na educação chilena, é provável que a pontuação obtida por eles na Prova de Seleção Universitária (PSU), não seja suficiente para o ingresso na universidade. Para estes jovens, algumas instituições têm implementado os Programas propedêuticos. Mas, qual é

* Este estudio forma parte de la investigación "Motivación y expectativas para el aprendizaje efectivo en estudiantes de enseñanza secundaria y universitaria, de contextos socioeconómicos vulnerables, que participan en Programa Propedéutico y Bachillerato en Educación Superior UCSH”. Financiada por CONICYT a través del proyecto de Inserción de Capital Humano en la Academia n 79100022. 
seu discurso em relação a estes programas? Por que obtêm bons resultados? Por meio da análise estrutural do discurso, evidenciam-se as características que os jovens têm. Características que, com o apoio necessário, poderiam torná-los candidatos capazes de completar com sucesso uma carreira universitária.

Palavras chave: programas propedêuticos, PSU, ranking, retenção, abandono.

\section{INTRODUCCIÓN}

El acceso a la educación superior y las desigualdades que en este sentido se producen, son un punto de discusión en todos los ámbitos de la esfera nacional. Para dar cuenta de esta situación, basta con señalar que mientras 3 de cada 4 alumnos pertenecientes al $20 \%$ más rico de los hogares chilenos accede a la educación superior, sólo 1 de cada 7 alumnos pertenecientes al quintil más pobre se encuentra en igual situación.

Una de las principales causas de este problema se atribuye al actual sistema de admisión basado en la Prueba de selección Universitaria (PSU). Se dice que este ha marcado las posibilidades de ingreso de los jóvenes a la universidad a partir de su origen socioeconómico y tipo de establecimiento de procedencia (Contreras, Corbalán y Redondo, 2007; Kremerman, 2007; OCDE, 2009). En efecto, el 43,2\% de los alumnos que rinde la PSU obtiene menos de 450 puntos. El 62\% de estos estudiantes provienen de colegios municipales y, por lo general, estos estudiantes quedan fuera de la educación universitaria (Universidad de Chile, 2011).

Otro problema que se presenta es que los resultados obtenidos en la PSU no sólo establecen la selección para el ingreso a la universidad, sino que además son un factor determinante para la distribución de créditos y becas, pues para optar a ellos se exige un mínimo de 475 puntos. Frente a esta situación Acuña y Arévalo et al. (2009), plantean que la PSU es un sistema segregador que reproduce las desigualdades sociales, ya que los estudiantes con mayores recursos también acceden a sistemas de preparación en preuniversitarios de alto costo, situación que aumenta la injusticia debido a que las familias de jóvenes con menores ingresos no pueden costear esta preparación.

Por su parte, Manzi (2012) sostiene que las inequidades no provienen de la PSU como sistema de selección para el ingreso a la educación superior, ya que esta prueba evidencia lo mismo que todas las mediciones que se aplican en Chile: señala que el rendimiento está estrechamente correlacionado con el nivel socioeconómico, por lo tanto, el problema no se encuentra en los test sino que en las profundas desigualdades de la sociedad nacional y en los sistemas de selección para ingresar a las universidades. El autor afirma que no se puede exigir equidad a las pruebas; la equidad debe estar en los sistemas de selección. Su propuesta incluye indicadores que no estén correlacionados con el origen social de los estudiantes, por ejemplo, el ranking de notas en el colegio de origen de los postulantes. Es por esta razón que Manzi propone como una vía válida de acceso a la universidad los Programas Propedéuticos.

Aún cuando no son un sistema oficial de ingreso a la universidad y pocas instituciones cuentan con este tipo de programas, los Propedéuticos son una vía alternativa para que estudiantes de contextos vulnerables, que se destacan por sus méritos académicos $(5 \%-10 \%$ superior en ranking) ingresen a la universidad sin considerar los puntajes obtenidos en la PSU. Se parte de la base de que estos jóvenes tienen altos promedios de notas y ello corresponde a una habilidad relativa que también es un predictor válido de éxito académico (Gallegos y Meneses, 2007; Contreras, Gallegos y Meneses, 2009; Meneses y Toro, 2012). 
Los estudios predictivos a partir del ranking se han realizado con jóvenes que ya son parte de las universidades y que, por lo tanto, han pasado la barrera PSU, sin embargo, se dispone de escasa información con respecto al perfil que tienen los estudiantes que son parte de los programas propedéuticos: ¿Qué características tienen estos jóvenes que los hacen destacarse por sus altas calificaciones? ¿Qué significa para ellos ser parte del programa propedéutico en la UCSH? Estas son las interrogantes principales que guían el presente trabajo. Para ello se describe a continuación algunas referencias que se han seleccionado para sustentar tanto análisis como resultados.

\section{MARCO DE REFERENCIA}

\subsection{INGRESO Y RETENCIÓN DE LOS ESTUDIANTES A LA EDUCACIÓN SUPERIOR}

En chile, a diferencia de otros países de Latinoamérica, para acceder a la educación superior los estudiantes son elegidos por las universidades a partir de los resultados en la prueba de selección universitaria (PSU). Ella consiste en la aplicación de una batería de test que miden habilidades cognitivas en Lenguaje y Comunicación, Matemática, Historia y Ciencias Sociales y Ciencias. El puntaje que los estudiantes obtienen se pondera con el promedio de notas de enseñanza media (NEM). Al NEM se le asigna una ponderación mínima de $20 \%$, según lo establecido por el Consejo de Rectores. El porcentaje restante se distribuye de acuerdo al valor que asigne cada universidad y carrera a los resultados de la PSU (Donoso y Schiefelbein, 2007).

Según Donoso y Schiefelbein, la instalación de un sistema de selección de estudiantes, por más de siete décadas, se sustenta en la premisa de que no todos los egresados de la enseñanza secundaria cuentan con las habilidades, condiciones, capacidades, aptitudes o competencias para concluir estudios universitarios. En consecuencia, el propósito del proceso selección es certificar un nivel mínimo de desempeño, para aumentar la posibilidad de su retención. Himmel (2002) define la retención como:

Persistencia de los estudiantes en un programa de estudios universitarios hasta lograr su grado o título. Esta categoría se puede limitar a la situación del estudiante que obtiene su título o grado en el tiempo mínimo establecido por la institución para completarlo, o bien, en forma más general, no considerar el tiempo que demore en alcanzarlo, debido a demoras por "repitencia", por suspensión de los estudios durante algunos períodos académicos o porque se comprometió con una carga académica menor que la establecida (2002: 4).

Si se considera los objetivos que tiene el sistema de selección de estudiantes para el ingreso a la universidad, aún no se cuenta con evidencia suficiente para asegurar que, tanto PSU, como NEM son los únicos y mejores predictores de retención en educación superior. En efecto, recientes investigaciones realizadas en Chile concluyen que aquellos estudiantes que obtienen los mejores promedios de notas en sus respectivos establecimientos (NEM y PSU), obtienen mejores desempeños en educación superior. En consecuencia, lo que proponen los autores es incorporar el sistema de Ranking como una vía de ingreso a la universidad (Gallegos y Meneses, 2007; Contreras, Gallegos y Meneses, 2009; Meneses y Toro, 2012).

Es por esta razón que a mediados del mes de junio de 2012, se ha difundido en los medios de comunicación que las universidades chilenas ponderarán el ranking de notas 
en el proceso de admisión 2013. El ranking consiste en ordenar a los alumnos según rendimiento en la enseñanza media, relativo a sus compañeros de egreso del mismo establecimiento escolar. Esta medida de habilidad relativa (o ranking) compara al alumno respecto de sus pares (que se desenvuelven en un mismo contexto y entorno), por lo que podría ser indicativa respecto de las proyecciones académicas del estudiante. Sin embargo, es posible que aunque el ranking efectivamente entregara dicha información, esta ya haya sido capturada por los elementos de la batería de selección universitaria.

En todo caso, existe cierto consenso en determinar que las características de los estudiantes, como habilidad relativa, juegan un rol importante en la continuidad y conclusión exitosa de los estudios, no obstante, también se debe tener en cuenta que el Informe sobre la repitencia y deserción en la educación superior chilena publicado por IESALC - UNESCO (2005), declara que las características de los modelos de docencia, en el sistema de selección y en el efecto combinado ambos, son factores explicativos fundamentales que llevan a los estudiantes a abandonar la universidad. Adicionalmente, se pueden considerar los factores económicos, particularmente en el caso de las universidades privadas, donde los estudiantes deben cubrir el costo de su educación, existiendo un apoyo económico muy limitado.

En consecuencia, también se debe tener presente que la retención no solo depende de variables atribuidas a los estudiantes. En este sentido, se sostiene que la deserción/ retención es el resultado de la combinación y efecto de distintas variables. En estas se encuentran características preuniversitarias, institucionales, familiares, individuales y las expectativas laborales. Estas influyen en la integración social y académica, las cuales a través del grado de motivación del estudiante provocan un efecto positivo -aumentando la probabilidad de permanecer- o negativo -presentando mayor probabilidad de desertar (Díaz, 2007).

Por su parte, Donoso y Schiefelbein (2007) consideran como variables de deserción, factores atribuidos a: (i) Características previas del sujeto y su éxito en los primeros años de universidad, (ii) Identificación de los aspectos a los cuales los estudiantes atribuyen importancia antes de graduarse, (iii) descripción de experiencias diseñadas para incrementar la retención. (iv) Análisis de resultados de procesos de innovación pedagógica y su impacto en la retención (como dimensión en pleno desarrollo).

En síntesis, si bien es cierto que las características de los estudiantes son relevantes para concluir con éxito la formación universitaria, las instituciones deben brindar un apoyo integral y diseñar experiencias de acompañamiento y formación los jóvenes, más aún cuando estos pueden encontrarse en desventaja social y económica al ingresar a la universidad. Esto significa dar un paso en cual las entidades refuerzan su rol de liderazgo social al interpretar la realidad y necesidades de sus estudiantes y establecen mecanismos que, además de considerar el acceso, también establezcan planes de retención y egreso oportuno.

2.2. LOS PROGRAMAS PROPEDÉUTICOS COMO CONTRIBUCIÓN E

N LA DISMINUCIÓN DE LA SEGREGACIÓN EN LA EDUCACIÓN SUPERIOR

La segregación en la educación superior no es un problema reciente. Ya en el año 1967 Pierre Bourdieu (2003) en su texto Los herederos sostenía que las posibilidades y oportunidades de elección de carreras que tienen los jóvenes al entrar a la universidad, según su clase social de origen, viene ya sorteada desde los primeros años de 
escolarización; según el autor, se aprecia que niños y niñas pertenecientes a niveles sociales menos favorecidos, se encuentran en absoluta desventaja en comparación con sus pares provenientes de clases sociales con mayores privilegios desde antes de ingresar a las instituciones de educación formal.

Bourdieu (2003) plantea que los estudiantes deben sortear una serie de obstáculos entre disposiciones objetivas (institución) y subjetivas (sujeto). En este sentido, en el nivel de enseñanza superior, la desigualdad inicial de las diversas capas sociales ante la educación, se evidencia en que ellas están muy desigualmente representadas. En consecuencia, el ingreso y permanencia en las universidades, perpetúa la selección y estratificación social puesto que se encubre las diferencias que existen entre los representantes de los diversos estratos sociales. Así, los estudiantes de nivel educativo mencionado se relegan o son relegados por la institución educativa para dar paso a los "herederos" de las clases sociales medias y altas.

Bourdieu (2003) intenta comprender este problema y lo explica a partir del concepto de "Don". El "Don" es entendido como una disposición innata de talentos para aprender y desenvolverse en las instituciones educativas, estos dones se desarrollan prioritariamente en el seno de la familia. En consecuencia y, desde esta perspectiva, los estudiantes de condiciones sociales más desfavorecidas vivirán la educación como una competencia injusta y como una conquista al no ser considerados portadores de dichos dones. En consecuencia, el ingreso a la universidad ha sido durante mucho tiempo un derecho exclusivo de las elites; un monopolio público financiado públicamente (Heras, 2009).

Esta situación también ha sido estudiada en América Latina con el fin de dar solución a las dificultades derivadas de la exclusión. Por ejemplo, diversos países de la región han desarrollado las llamadas Políticas de Acción Afirmativa. Estas políticas buscan concretar el principio de igualdad material y la neutralización de las consecuencias de la discriminación racial, de género, de origen nacional, de edad y de complexión física (Sverdlick, Ferrari y Jaimovich, 2005). No obstante, los autores plantean que las acciones que se han implementado no han significado una modificación sustancial de las características elitistas de la educación superior. "Son, en este sentido, sistemas de educación superior excluyentes en sociedades que también son excluyentes" (2005: 109).

Si se considera la realidad chilena, Kremerman (2007) plantea que la cobertura en educación superior ha aumentado en los últimos 15 años. Al comparar las cifras entregadas por la encuesta CASEN 1990 y 2003, se puede observar que se detecta un alza de más de 20 puntos porcentuales, pasando de $16,0 \%$ a 37,5\%. Por su parte, el gasto en Educación Superior en Chile es uno de los más altos en el mundo como porcentaje del Producto Interno Bruto. En Chile se gasta casi un 2,2\% del PIB en Educación Superior, 0,8 puntos porcentuales más que el promedio de los países pertenecientes a la OCDE, siendo sólo superado por Corea del Sur.

Sin embargo, dado que en el contexto nacional la estructura de educación superior es altamente privatizada, la mayor parte de los recursos que ingresan al sistema proviene de las familias y de los propios alumnos y se canaliza a través de una modalidad descentralizada y dirigida a insumos (Brunner, 2007). Como resultado, aquellos que no cuenten con estos recursos están en peligro de quedar excluidos y la exclusión puede comprenderse como "factores de vulnerabilidad que genera el proceso de acumulación de desventajas, proceso que podría derivar en una fractura del lazo social" (Donoso \& Schiefelbein, 2007: 12). En este contexto, los estudiantes pueden romper su vinculo social 
con la educación y se estaría, prácticamente sin discusión, ante exclusión educativa en la educación superior, sin importar el rendimiento académico que hayan obtenido a lo largo de sus estudios secundarios.

A partir de lo anterior, se fundamenta la creación de los Programas Propedéuticos. Estos programas tienen como propósito incluir a los estudiantes de contextos vulnerables, que presentan alto rendimiento académico (promedio de notas), en sus respectivos establecimientos en la educación superior, sin considerar los resultados obtenidos en la PSU.

La institución pionera en la implementación de este tipo de programa, en el año 2007, es la Universidad de Santiago de Chile (USACH). Posteriormente, en el año 2009 se adhieren a esta iniciativa La Universidad Católica Silva Henríquez y la Universidad Alberto Hurtado (UAH). Finalmente, a partir del año 2010, esta vía de acceso a la educación superior es implementada por la Universidad Tecnológica Metropolitana (UTEM), Universidad Metropolitana de Ciencias de la Educación (UMCE) y la Universidad Católica de Norte (UCN) sede Coquimbo, totalizando así 6 universidades. Todos estos planteles constituyen en la actualidad la "Red de universidades Propedéutico UNESCO". En términos generales, los programas propedéuticos siguen las siguientes directrices:

- Invitación y selección de los estudiantes que pertenecen al 5\% o 10\% superior en promedio de notas (ranking) en sus respectivos establecimientos.

- Clases de nivelación durante el segundo semestre los días sábados a cursos de Lenguaje y comunicación, matemáticas y gestión personal

- Los estudiantes que cursan el Programa propedéutico tienen la posibilidad de ingresar al programa de Bachillerato, y luego de un año, ingresar a una carrera universitaria.

Algunos de los principios orientadores de la acción de los Propedéuticos son los siguientes (ver www.propedeutico.cl):

- Los talentos están igualmente distribuidos entre ricos y pobres.

- La educación es una herramienta de fuerte movilidad social, además de proveer mayores oportunidades de desarrollo personal y de convivencia democrática.

- Las notas de educación media son un mejor predictor de rendimiento académico que las pruebas nacionales de selección como la PSU.

- La PSU replica las condiciones de inequidad y exclusión social.

- Hablar de acceso a la educación superior es incompleto, pues la verdadera justicia social se produce al egreso. Se deben aumentar los programas de apoyo que eviten la deserción de los estudiantes, así como respetar las particularidades socio-cultural de éstos.

- Los programas de acción afirmativa son necesarios para trabajar situaciones de exclusión social en condiciones de vulnerabilidad.

- El sentir y palpar oportunidades reales de desarrollo personal actúan como una gran potencia motivadora.

- Personas que provienen de medios vulnerables requieren un período de tiempo para nivelar resultados académicos. Estas personas presentan indicadores de retención mayor a otros grupos.

También es posible valorar el aporte de estos programas en la retención universitaria ya que como plantea Díaz (2008) la transición de jóvenes desde la enseñanza media a la educación es un proceso que resulta dificultoso, más aún en el caso de aquellos jóvenes que provienen de contextos sociales menos favorecidos ya que ellos se encuentran en desventaja en comparación a sus pares provenientes de estratos superiores. 
Sin embargo, reconociendo el aporte que en materia de oportunidad de acceso a educación son los programas Propedéuticos, también es importante considerar otras opiniones, por ejemplo, Acuña y Arévalo et al. (2009), sostiene que os programas propedéuticos enmascaran barreras estructurales que se presentan el sistema social y educativo.

No obstante, atendiendo a lo anterior, en este texto se reconoce que el Propósito de los Programas Propedéuticos no es solucionar los problemas sociales con una acción, sino que más bien ofrecer una alternativa a acceso a la universidad a aquellos jóvenes que se destacan por su rendimiento académico.

Es evidente que recién se está comenzando en este camino, pero acciones como las que se han descrito son un aporte en materia de respeto por las diferencias y promoción de valores asociados a la justicia social. Sin duda, es importante seguir desarrollando acciones que otorguen mejores respuestas y oportunidades. De ahí la relevancia de la investigación en este campo y la sistematización de la información, de manera que a partir de ella se genere conocimiento sólido que permita el diseño de políticas públicas que aseguren que todos los sectores sociales estén representados en la educación superior.

Es por ello que desde el año 2009 la modalidad de acceso vía propedéutico ha sido incorporada por la Universidad UCSH. En la búsqueda del enriquecimiento y fortalecimiento se investiga y analiza el programa a través del proyecto Motivación y expectativas para el aprendizaje efectivo en estudiantes de enseñanza secundaria y universitaria, de contextos socioeconómicos vulnerables, que participan en Programa Propedéutico y Bachillerato en Educación Superior UCSH, pues tal como sostiene Mersten (1999), el papel de la investigación debe apuntar hacia cambios sociales justos a favor de aquellos/as que son discriminados. Sus voces proporcionan más credibilidad ya que la transformación sólo puede ocurrir si su información es usada para fundamentar programas sociales que intentan atajar las desigualdades existentes.

\section{METODOLOGÍA}

En su fase cualitativa se considera que la orientación de investigación evaluativa como una acción social, contextualizada, política, ética y orientada por valores en donde todos los actores deben participar, sus juicios y valoraciones deben ser incorporados, especialmente aquellas voces que han sido ignoradas (Mersten, 1999; Stake, 1998; Eisner, 1991; Pattom, 1990; Guba y Lincoln, 1989).

Se opta por esta perspectiva pues se considera que la investigación evaluativa no tiene como único centro la recolección de datos y la descripción del funcionamiento de programas. Desde una perspectiva crítica, por ejemplo, se comprende como un proceso que fomenta la reflexión de las circunstancias personales, sociales, políticas y económicas que rodean la acción.

Como técnica de recolección de información se ha utilizado la entrevista en profundidad, ya que esta técnica tiene como propósito central encontrar lo que es significativo e importante en la mente de los informantes, sus perspectivas, interpretaciones, el modo en que ellos ven, clasifican y experimentan su propio mundo (Ruiz, 2003).

Con posterioridad a la especificación de las características de los actores que contribuirían en la entrega de información, se construyó un protocolo de entrevista en el cual se establecieron los objetivos específicos y las dimensiones a cubrir. A partir de éstos, se 
levantaron preguntas que cubren estos ejes y objetivos. Luego, estas preguntas se revisaron y sometieron a juicio de expertos. Se realizó un nuevo ajuste a las preguntas y se construyó un protocolo definitivo de aplicación. Este protocolo se utilizó en cuatro entrevistas y todas las dimensiones y ejes fueron cubiertos. Asimismo, todas las preguntas fueron comprendidas sin dificultad y se obtuvo a partir de ellas la información que se buscaba.

Para el estudio de los discursos se utilizó el análisis contenido estructural, siguiendo las orientaciones de Martinic (1995; 2006). Según el autor, este tipo de análisis se basa en un procedimiento inspirado en la lingüística estructural, que permite identificar, en primer momento, las unidades básicas de sentido y las relaciones existentes entre ellas y en un segundo momento, el método organiza el movimiento de tales relaciones en un modelo de acción social (modelo actancial) que orienta la práctica de los sujetos enunciadores del discurso.

Los resultados recomponen las estructuras, identificando las realidades o temas que se asocian entre sí a través de relaciones de oposición o equivalencia. Luego se dinamizan las estructuras, distribuyendo las oposiciones y asociaciones identificadas en un modelo de acción social. Finalmente, se analizan las funciones simbólicas que asumen las distintas realidades o elementos considerados en el paso anterior, asumiendo que dicha organización y estructura da sentido a las prácticas o acciones que realizan los sujetos.

\section{RESULTADOS}

A partir del análisis realizado a las entrevistas de los estudiantes del programa propedéutico se han construido los códigos de oposición. Estos códigos presentan el principio simbólico subyacente en sus discursos: "Ser un profesional". Esto constituye una identidad positiva en los entrevistados. Para cumplir con este principio, la educación en la universidad, adquiere una valoración relevante y se opone a "ser un trabajador" ya que esta situación se asocia con personas que están fuera de la universidad o no estudian en ella.

Ser profesional (+): En la universidad, buen sueldo, con conocimientos, feliz, tener familia, ser un aporte. Orgulloso de mí mismo.

Ser un trabajador (-): fuera de la universidad, pobre, ignorante, frustrado, aproblemado, ser una carga.

\subsection{PROTOCOLO ANALÍTICO ESTRUCTURA PARALELA}

La estructura paralela presenta la organización que tiene la representación que los estudiantes entrevistados tienen sobre los sistemas de selección para el ingreso a la universidad. Los códigos de calificación atribuyen propiedades de un modo sistemático paralelo e inverso a los términos o realidades que forman el código de base.

En este caso, la totalidad de calificación corresponde a los sistemas de selección para ingresar a la universidad y los códigos de base corresponden a la PSU con una valoración negativa y al programa propedéutico con una valoración positiva.

En los discursos analizados, la exigencia PSU demanda altos puntajes. Esta situación dificulta las posibilidades de ingreso a la universidad ya que el éxito solo se basa en estos resultados. En consecuencia, esta modalidad de selección se asocia y valora como un sistema segregador para los estudiantes que pertenecen a contextos vulnerables. En consecuencia, esto implica una limitación del sistema educativo en su conjunto. 
Figura 1. Estructura paralela*

\begin{tabular}{|c|c|c|c|c|}
\hline & $(+)$ & $\mathbf{T}$ & $(-)$ & \\
\hline \multicolumn{5}{|c|}{ VIASSE INGRESO A LA EDUCACIÓN SUPERIOR } \\
\hline C06 & $\begin{array}{c}\mathrm{A} 1 \\
\text { A vance y democracia }\end{array}$ & l & $\begin{array}{c}\text { B1 } \\
\text { Limitación }\end{array}$ & $\begin{array}{c}\text { TC1 } \\
\text { Impacto }\end{array}$ \\
\hline & & & & \\
\hline $\begin{array}{l}\text { CC5 } \\
\text { II }\end{array}$ & $\begin{array}{c}\mathrm{A} 2 \\
\text { "No hay segregación con los otros } \\
\text { alumnos y la universidad confía en } \\
\text { esos alumnos" }\end{array}$ & I & $\begin{array}{c}\text { B2 } \\
\text { (Segregador para los estudiantes de } \\
\text { contextos vulnerables) }\end{array}$ & $\begin{array}{c}\text { TC2 } \\
\text { Características del } \\
\text { sistema }\end{array}$ \\
\hline & & & & \\
\hline II & $\begin{array}{c}\text { A3 } \\
\text { "Creo que la oportunidad de } \\
\text { estudiar gratis y hay que } \\
\text { aprovecharla" }\end{array}$ & l & $\begin{array}{c}\text { B } 3 \\
\text { La oportunidad de estudiar gratis } \\
\text { debería beneficiar a más estudiantes. }\end{array}$ & $\begin{array}{c}\text { TC3 } \\
\text { Beneficios } \\
\text { económicos }\end{array}$ \\
\hline & & & & \\
\hline $\mathrm{CC3}$ & $\begin{array}{c}\text { A } 4 \\
\begin{array}{c}\text { Pocas, porque el éxito depende del } \\
\text { estudiante }\end{array} \\
\end{array}$ & 1 & $\begin{array}{c}\text { B } 4 \\
\text { M uchas, porque el éxito depende de los } \\
\text { resultados PSU }\end{array}$ & $\begin{array}{c}\text { TC4 } \\
\text { Dificultades }\end{array}$ \\
\hline & & & & \\
\hline II & $\begin{array}{c}\text { A5 } \\
\text { B uena oportunidad de selección para } \\
\text { acudir a la universidad (notas). }\end{array}$ & $T$ & $\begin{array}{c}\mathrm{A5} \\
\text { Dificultades para ingresar }\end{array}$ & $\begin{array}{c}\text { TC } 5 \\
\text { Oportunidades de } \\
\text { ingreso a la } \\
\text { universidad }\end{array}$ \\
\hline & & & & \\
\hline $\begin{array}{l}\text { CC1 } \\
\pi\end{array}$ & $\begin{array}{c}\text { A6 } \\
\text { Baja } \\
\end{array}$ & I & $\begin{array}{r}\text { B6 } \\
\text { Alta } \\
\end{array}$ & $\begin{array}{c}\text { TC6 } \\
\text { Puntajes }\end{array}$ \\
\hline $\begin{array}{l}\text { CB } \\
\text { II }\end{array}$ & $\begin{array}{c}\mathrm{A} \\
\text { Propedéutico }\end{array}$ & 1 & $\begin{array}{c}\text { B } \\
\text { PSU }\end{array}$ & $\begin{array}{l}\text { Sistemas de } \\
\text { selección } \\
\text { Universitaria }\end{array}$ \\
\hline
\end{tabular}

En donde

\begin{tabular}{|l|l|l|}
\hline cAcB & $=$ & Realidades o términos de calificación \\
\hline $\mathrm{A}, \mathrm{B}$ & $=$ & Términos o realidades o objetos significantes \\
\hline $\mathrm{cc}$ & $=$ & Códigos de calificación o implicación \\
\hline $\mathrm{cb}$ & $=$ & Código de base \\
\hline $\mathrm{Tc}$ & $=$ & Totalidad calificativa \\
\hline $\mathrm{Tb}$ & $=$ & Totalidad de base \\
\hline $\mathbf{I}$ & $=$ & Implicación lógica \\
\hline
\end{tabular}

*A míme beneficia por la PSU, porque yo igual no estoy preparada para una buena PSU, me falta mucho creo yo (Stephanie). Creo que es una gran oportunidad para las personas que tienen bajo recursos. Se tienen que esforzar muchísimo para salir adelante y es una ventana más que se le abre a todos los jóvenes como yo (Thomas). Considero que todas las universidades de Chile debiesen tener propedéutico, porque lo considero, educacionalmente, es como una democracia que se abre, le da la opción a toda clase de persona de entrar a la universidad. Considero que es un avance que se ha hecho en la educación (Kain).

Por su parte, el Programa Propedéutico y Bachillerato es definido como un sistema que exige bajos puntajes y prioriza la selección a través de las notas obtenidas en la enseñanza media. Esta situación es valorada como una buena oportunidad para ingresar a la universidad ya que disminuye las barreras derivadas de las exigencias de la PSU debido a que las calificaciones obtenidas son atribuidas a las características del estudiante, por lo tanto, con estas acciones la universidad es representada como un sistema que no excluye a las personas que provienen de colegios vulnerables y que, por diversas razones, no han tenido la preparación adecuada para la prueba. En consecuencia, para 
los entrevistados, los programas Propedéuticos son un reflejo de sistema educativo más democrático, en consecuencia, "todas las universidades" deberían contar con un sistema de selección como éste.

En síntesis se aprecia que los sistemas universitarios, con sus políticas de acceso, son representados como sistemas complejos, con altos niveles de segregación en los cuales, además, existe una gran inequidad escasas oportunidades para aquellos que presentan carencia de recursos económicos.

Figura 2. Estructura Cruzada ${ }^{\circ}$ 1: Actitud/calificaciones**

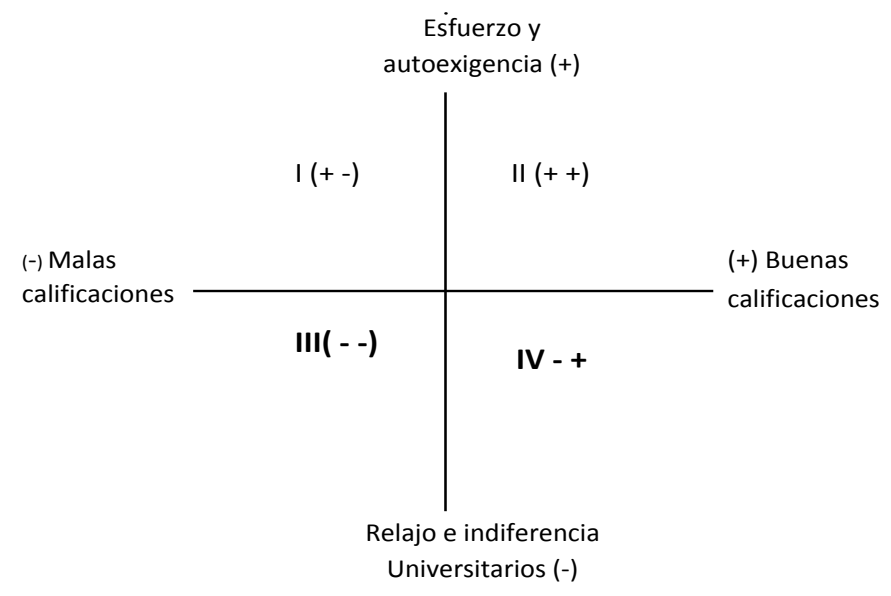

\begin{tabular}{|l|l|}
\hline I & Estudiantes que se esfuerzan y se autoexigen con malas calificaciones \\
\hline II & Estudiantes que se esfuerzan y se autoexigen con buenas notas \\
\hline III & Estudianes que se relajan y son indiferentes con malas calificaciones \\
\hline
\end{tabular}

**Los compañeros nos pasan a llevar a nosotros que sí íbamos al colegio, sí nos sacábamos buenas notas, y no nos beneficiaba en "nada"; podíamos hacer lo que queríamos, igual íbamos a pasar. También tengo compañeros, yo creo que también no tenían el promedio para entrar acá, porque se necesitaba un promedio alto en toda la enseñanza media para entrar, era un requisito (Stefanie). Soy una persona esforzada, autoexigente conmigo mismo, lo cual siempre me hace ir más arriba (Thomás).

\subsection{PROTOCOLO ANALÍTICO ESTRUCTURA CRUZADA $\mathrm{N}^{\circ}$ 1: ACTITUD/CALIFICACIONES}

Los estudiantes que ingresan al programa propedéutico obtienen el 5\% superior en promedios de notas. En este sentido, los jóvenes entrevistados declaran que este rendimiento se debe a su esfuerzo y autoexigencia (campo semántico $\mathrm{n}^{\circ} \mathrm{II}$ ). Marcan la diferencia en sus acciones y actitudes de las que realizan otros compañeros que no se toman con seriedad los estudios y que, más bien, son una barrera para el aprendizaje de aquellos que sí desean progresar y aprender. El grupo al que aluden obtienen malas calificaciones producto de su relajo e indiferencia (campo semántico $\mathrm{n}^{\circ}$ III). No obstante, también en sus discursos se hace mención a aquellos pares que aún cuando se esfuerzan 
y se autoexigen, no logran obtener buenos resultados (campo semántico $\mathrm{n}^{\circ} \mathrm{I}$ ). Este caso da cuenta de aquellos jóvenes que serían los menos beneficiados ya que sus oportunidades son incluso más limitadas. En el campo IV se encuentran aquellos estudiantes que se relajan son indiferentes y obtienen buenas calificaciones. Sin embargo, es importante destacar que esta calificación no se encontró en los discursos que hasta el momento se han analizado.

Figura 3. Estructura cruzada Tipos de familia/Apoyo***

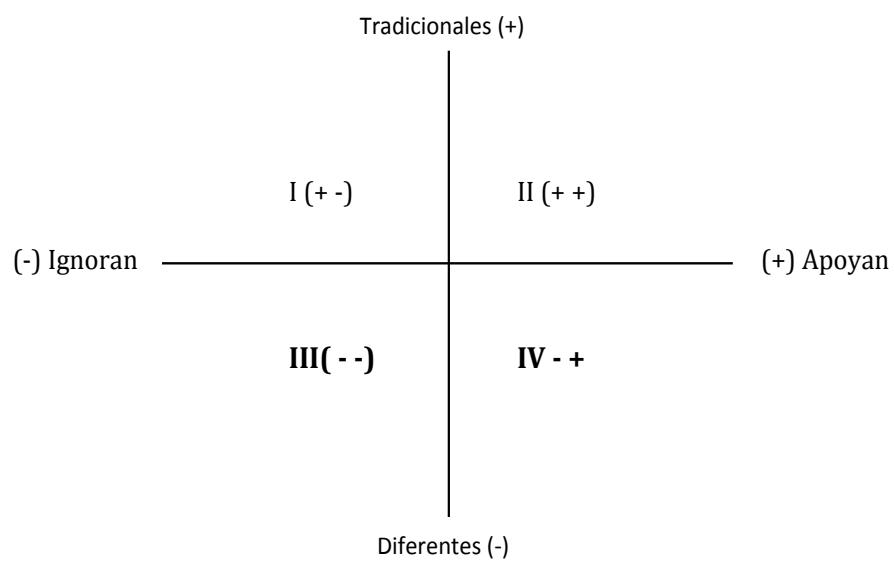

\begin{tabular}{|l|l|}
\hline I & Familias tradicionales que ignoran a sus hijos \\
\hline II & Familias tradicionales que apoyan a sus hijos \\
\hline III & Familias diferentes que apoyan a sus hjos \\
\hline IV & Familias diferentes que ignoran a sus hijos \\
\hline
\end{tabular}

\footnotetext{
***No sé como que estén al lado mío, no sé, con eso estoy bien, a veces no sé, se preocupan. Me retan, pero no importa, como que hasta a veces pienso que no toman mucha atención (Stephanie). Me fui de lado de mi familia porque no compartía las mismas ideas que el resto. No les importo (Kain). Mi mamá no podía hacer cosa de padre. Por eso, pero ahora que tengo otra visión de vida creo que fue mejor, la mejor opción fue que mi mamá haya tomado ese rol de madre y padre... Mi mamá para sacarme adelante trabajaba siempre, trabajaba todos los días. Tenía turnos desde las ocho hasta la noche (Thomas).
}

\subsection{PROTOCOLO ANALÍTICO ESTRUCTURA CRUZADA N ${ }^{\circ} 2$ : ESTRUCTURA CRUZADA TIPOS DE FAMILIA/APOYO}

Cuando se analizan las calificaciones respecto de las familias, se aprecia que la valoración más positiva se realiza en aquellas situaciones en las cuales las familias tradiciones (papa, mamá, tíos, hermanos) apoyan el proceso y los estudios (campo semántico $\mathrm{n}^{\circ}$ II). Por otra parte, también son una realidad presente en los discursos, aquellas familias 
consideradas tradicionales que son indiferentes, sin embargo, a pesar de esta indiferencia, son bien valoradas por los jóvenes ya que su sola presencia es una motivación para los estudios (Campo semántico $\left.\mathrm{n}^{\circ} \mathrm{I}\right)$.

También están presentes las familias que siendo diferentes (sólo madre, por ejemplo) son bien valoradas ya que los apoyan y motivan para que continúen con sus esfuerzos y sean un ejemplo para sus hermanos (Campo semántico ${ }^{\circ}$ IV). Finalmente, están aquellas familias diferentes (en valores, creencias y constitución) que ignoran y restan valor al desarrollo académico (campo semántico $\mathrm{n}^{\circ} \mathrm{III}$ ). En este caso, los jóvenes prefieren desvincularse de ellas y, en algunos casos, es un logro abandonarlas y salir de su lado en la búsqueda de un camino que los lleve hacia el éxito y la realización de ser profesional.

Figura 4. Modelo de acción****

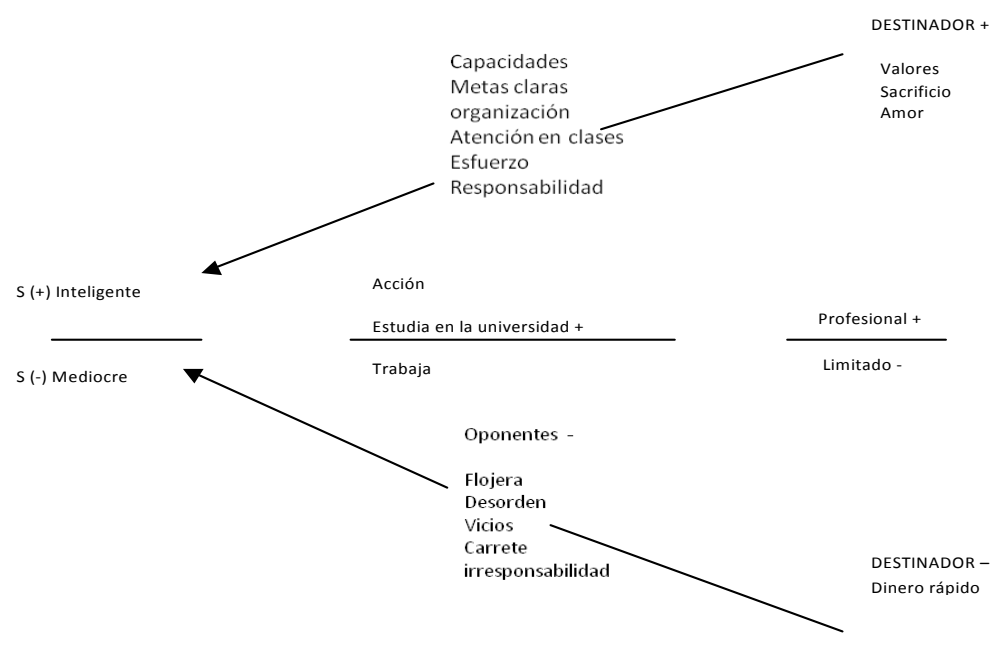

\footnotetext{
****Creo que, de repente, cuando la gente dice que esta persona es más inteligente que esta, yo creo que están equivocados porque, yo creo, no sé qué es lo que piensa Ud., pero yo creo que todos nacemos con la misma inteligencia, con la misma caja de herramientas, solamente hay personas que esas herramientas algunos tienen capacidades de poder ejercerlas en su vida más bien que otras (Thomas). Tengo mis metas claras, me organizo, tomo atención en clases, me tomo como con responsabilidad las cosas que tengo que hacer y lo tengo que hacer bien (Stephanie). Soy una persona bien esforzada, cercano con mis profesores, soy sacrificado. Soy un niño casero, como se dice, no me gusta andar; o sea me gusta salir a fiestas de repente, de vez en cuando. Estudio y tengo facilidad también para aprender (Kain).
} 


\subsubsection{La búsqueda del bien deseado. Relación sujeto-objeto}

Este eje está definido por la alternativa existencial del sujeto. La realidad está percibida de acuerdo a la dicotomía ser inteligente/mediocre. De esta forma se construye el objeto positivo y negativo respectivamente. Cuando este sujeto corresponde a un objeto positivo, se transforma en un profesional. Cuando el sujeto mediocre se relaciona con un objeto negativo, se transforma en un limitado.

\subsubsection{Las acciones para llegar al objeto positivo}

Para los jóvenes entrevistados, un sujeto inteligente se transforma en el objeto deseado o profesional cuando realiza la acción de estudiar en la universidad. El efecto contrario se produce cuando un sujeto mediocre realiza la acción de trabajar hecho que convierte al sujeto en un objeto o persona limitada.

\subsubsection{Los ayudantes y oponentes para llegar al objeto}

Las ayudas que contribuyen a alcanzar el objeto deseado (ser profesional), adquieren valores positivos si ellas permiten alcanzarlo o bien tienen el efecto contrario. En este caso es posible identificar que, para ser un profesional es necesario un sujeto inteligente, pero además se requiere de capacidades que se traducen en tener metas claras, organización, atención en clases, esfuerzo, responsabilidad y capacidades para aprender. En oposición, se encuentra la flojera, el desorden, los vicios, el carrete (fiestas, diversión) e irresponsabilidad.

\subsubsection{La comunicación del destinador hacia el destinatario}

Aquí se presentan los elementos que comunican u otorgan al sujeto un poder para desencadenar o avalar su acción. Estos elementos asumen la función de comunicar una fuerza o mensaje para que este lleve a cabo la acción de estudiar en la universidad y ser profesional. Este mensaje es comunicado al destinatario, quien legitima su actuar asumiendo un proyecto que va más allá de su cotidianeidad. A partir de lo planteado por los estudiantes, el poder que desencadena sus acciones corresponde los valores éticos y morales de los cuales se consideran portadores. Asimismo, avala y sustentan sus acciones el sacrificio que realizan para obtener buenas calificaciones y distinguirse y destacarse en sus contextos. Todo ello, para responder al amor que sienten por sus familias o para cambiar la realidad (de abandono) desde la cual provienen. En oposición, se aprecia que la ambición económica es el principal motor que moviliza la conducta de los jóvenes considerados mediocres ya que en vez de hacer los sacrificios y esfuerzos que corresponden (desde sus juicios) prefieren mantenerse en sus contextos, reproducir el sistema y abandonar la escuela. 


\section{CONSIDERACIONES FINALES}

En la actualidad se está ante un escenario en el cual una mayor cantidad de jóvenes accede a la educación superior, al tiempo que otra parte importante de ellos queda totalmente excluida.

Esta situación es percibida y vivida por los jóvenes del Programa Propedéutico y Bachillerato de la UCSH, sin embargo, estos jóvenes se representan y presentan como un tipo diferente de estudiante. Un estudiante que se distingue del resto pues sus méritos y dones no se asocian a la clase social de la cual provienen. Ellos son herederos de esfuerzo, sacrificio, planificación y metas claras. Estas características son, entre otras, las acciones que los llevan a obtener buenas calificaciones, por lo tanto, detrás de la habilidad relativa o ranking descrita por Gallegos y Meneses (2007); Contreras, Gallegos y Meneses (2009); Meneses y Toro (2012), como buen predictor del éxito académico, se encuentran personas que han logrado ser los mejores a partir de sus propios medios, en contextos de alta vulnerabilidad.

Son herederos de familias de escasos recursos que, en algunos casos los apoyan, pero que en otros se desentienden de su educación. Aún así, están dispuestos a convertir los obstáculos en oportunidades de cambio y abandonar sus contextos si es necesario. No están dispuestos a relegarse y en sus discursos expresan el objetivo de ser un profesional, no obstante, la PSU aparece como un obstáculo en la realización de un sujeto positivo, debido a que reconocen que la preparación que han tenido en sus establecimientos no les permitirá obtener un buen puntaje (Contreras, Corbalán y Redondo, 2007; Krmerman, 2007; OCDE, 2009). En consecuencia, lo peor que les podría ocurrir es tener que abandonar la continuidad de sus estudios e ingresar al mundo laboral.

Aunque valoran los programas propedéuticos como un acto de democracia, de fondo también se encuentra impreso en sus discursos la valoración de estos programas como un acto de buena voluntad de parte de las universidades. En este caso, pasa a segundo plano todo aquello que los hace merecedores de alternativas de ingreso que consideren sus trayectorias educativas, es decir, aún cuando valoran positivamente las calificaciones que han obtenido y describen con elocuencia sus características y habilidades no cognitivas (esfuerzo, sacrificio, perseverancia, metas claras), se aprecia escasa conciencia de que son reales merecedores de ingreso a la universidad. Esto se evidente, ya que sus discursos tienen como centro la oportunidad económica (estudiar gratis) y con baja exigencia PSU que otorga el programa. Es decir, "se les da la oportunidad" en vez de "merezco la oportunidad".

Dicho lo anterior, es decir, que se merecen y se les debe dar la oportunidad de ingreso a educación superior a jóvenes que de otro modo hubiese quedado excluidos de estudiar en la Universidad, se levanta la voz de los jóvenes que señala que "Todas las universidades chilenas deberían tener Programa Propedéutico como vía de acceso alternativa a la PSU”. Frente a esta situación es prácticamente imposible dejar de cuestionarse lo siguiente: ¿cuántos jóvenes que presentan estas características queda totalmente excluido de la educación superior? ¿Cuáles son las ayudas y apoyos específicos que reciben los estudiantes una vez que entran a la universidad? ¿De qué forma y con qué estrategias compensa la universidad los costos personales que los jóvenes realizan para ingresar y permanecer en la institución? 
Se trata de considerar las propuestas de Díaz (2007) y Donoso y Schiefelbein (2007) y, además, de diseñar alternativas y políticas públicas concretas y reales de acceso a la universidad para jóvenes que pertenecen al 5\% superior de ranking, ya que ellos por derecho propio deben ingresar a la educación superior. Por lo tanto, se ha dado un paso al considerar el ranking en la ponderación de ingreso a la universidad para el año 2013.

Pero también se trata de un trabajo conjunto y organizado en pro de la retención y egreso oportuno de estos jóvenes, en el que las universidades ponen en práctica experiencias y acciones para fortalecer la integración social y académica, pues no se trata solo de que haya más cupos disponibles, sino políticas universitarias más inclusivas que respondan a las composición y características de su estudiantado.

\section{REFERENCIAS BIBLIOGRÁFICAS}

Acuña, F., Arévalo, C. et al. (2009). Acceso a la educación superior: el mérito y la (re) producción de la desigualdad. Santiago de Chile: Grupo de investigación CESCC - OPECH.

Bourdieu, P \& Passeron, J. (2003). Los herederos: los estudiantes y la cultura. Buenos Aires: Siglo XXI.

Brunner, J. J. (2007). Mercados universitarios: el nuevo escenario de la educación superior. Santiago: Universidad Diego Portales.

Contreras, A. Corbalán, F., y Redondo, J. (2007). Cuando la suerte está echada: estudio cuantitativo de los factores asociados al rendimiento en la PSU. Revista Electrónica Iberoamericana sobre Calidad, Eficacia y Cambio en Educación, vol. 5, n. 5 e.

Contreras, D., Gallegos, S. y Meneses, F. (2009). Determinantes de desempeño universitario: ¿importa la habilidad relativa? Análisis ex-ante y ex-post de una política pública. Informe final. Santiago: CNE.

Díaz, C. (2008). Modelo conceptual para la deserción estudiantil universitaria chilena. Estudios pedagógicos, $n$. 22, 65-86.

IESALC - UNESCO (2005). Estudio sobre la repitencia y deserción en la educación superior chilena. Santiago de Chile: IESALC - UNESCO.

Donoso, S. y Schiefelbein, E. (2007). Análisis de los modelos explicativos de retención de estudiantes en la universidad: una visión desde la desigualdad social. Estudios pedagógicos, vol. 22, n. 1, 7-27.

Eisner, E. W. (1991). The art of educational evaluation. London: The Falmer Press.

Gallegos, S. y Meneses, F. (2007). ¿Es Eficiente el Sistema de Ingreso a la Universidad? El uso del ranking en la universidad Católica de Chile. Web: http://www.ideaseneducacion.cl/wp-content/ uploads/2008/07/paper-puc-sistema-de-ingreso3.pdf

Guba, E. \& Lincoln, Y. (1989). Fourth Generation Evaluation. Newbury Park, CA: Sage.

Himmel, E. (2002). Modelos de Análisis para la deserción estudiantil en la educación superior. Calidad de la educación, $2^{\circ}$ semestre, 91-107.

Heras, H. (2009). Exclusión social en la educación superior chilena: programas y políticas para la inclusión. Tesis. Santiago de Chile: Universiteit Leiden.

Kremerman, M. (2007). El desalojo de la universidad Pública. Santiago: OPECH.

Manzi, J. (2012). ¿Equidad en las pruebas o en la admisión? Diario El Mercurio. Web: http:// blogs.elmercurio.com/cronica/2012/01/04/equidad-en-las-pruebas-o-en-la.asp (10/05/2012).

Martinic, S. (1995). Principios culturales de la demanda social por educación. Un análisis estructural. Pensamiento Educativo, n. 16, 313-39.

Martinic, S. (2006). El estudio de las representaciones y el análisis estructural del discurso. En Canales, M. (Ed.), Metodologías de investigación social (pp. 299-320). Santiago: Lom. 
Meneses, F. y Toro, J. (2012). Predicción de notas en Derecho de la Universidad de Chile: ¿sirve el ranking? Revista Isees, $n$. 10, 43-58.

Mertens, L. (1999). Labour Competence: emergence, analytical frameworks and institutional models. Montevideo: CINTERFOR/OIT.

OCDE (2009). Revisión de políticas nacionales de educación: la Educación Superior en Chile. Santiago de Chile: MINEDUC.

Patton, M. (1990). Qualitative evaluation and research methods. London: Sage.

Ruiz, J. (2003). El Diseño Cualitativo. Web: http://books.google.es/books?id=WdaAt6ogAykC\&pg $=$ PA51\&hl=es\&source $=g b s \_t o c \_r \& c a d=4 \# v=$ onepage $\& q \& f=$ false $(12 / 08 / 2010)$.

Sandín, M. (2003). Investigación Cualitativa en educación. Madrid: Mcgraw-Hill / Interamericana.

Stake, R. (1998). Investigación con Estudio de casos. Madrid: Morata.

Sverdlick, I., Ferrari, P. y Jaimovich, A. (2005). Desigualdad e inclusión en la educación superior. Un estudio comparado en cinco países de América Latina. Buenos Aires: PPCOR-OLPED.

Universidad de Chile. (2011). Compendio estadístico proceso de admisión año 2011. Santiago de Chile: Vicerrectoría de Asuntos Académicos, Departamento de Evaluación, Medición y Registro educacional. 